\author{
Xavier Delabranche \\ Julie Boisramé-Helms \\ Pierre Asfar \\ Asaël Berger \\ Yoganaden Mootien \\ Thierry Lavigne \\ Lélia Grunebaum \\ François Lanza \\ Christian Gachet \\ Jean-Marie Freyssinet \\ Florence Toti \\ Ferhat Meziani
}

\title{
Microparticles are new biomarkers of septic shock-induced disseminated intravascular coagulopathy
}

T. Lavigne

EA 4438, Faculté de Médecine,

Université de Strasbourg,

Strasbourg, France

L. Grunebaum

Laboratoire d'hématologie et Hémostase,

Hôpital de Hautepierre, Hôpitaux

Universitaires de Strasbourg,

Strasbourg, France

The online version of this article

(doi:10.1007/s00134-013-2993-x) contains supplementary material, which is available to authorized users.

F. Lanza - C. Gachet
X. Delabranche · J. Boisramé-Helms .

A. Berger - F. Meziani ( $)$

Service de Réanimation Médicale, Nouvel

Hôpital Civil, Hôpitaux Universitaires de

Strasbourg, 1, Place de l'Hôpital,

67091 Strasbourg Cedex, France

e-mail: ferhat.meziani@chru-strasbourg.fr

Tel.: +33-3-69550434

Fax: +33-3-69551859

X. Delabranche · J. Boisramé-Helms ·

A. Berger · F. Meziani

EA 3072, Fédération de Médecine

Translationnelle de Strasbourg (FMTS),

Faculté de Médecine, Université de

Strasbourg, Strasbourg, France

P. Asfar

Service de Réanimation Médicale et Médecine Hyperbare, Centre Hospitalier Universitaire d'Angers, Angers, France

Y. Mootien

Service de Réanimation Médicale, Centre Hospitalier de Mulhouse, Mulhouse, France
UMR S949 INSERM,

Université de Strasbourg et Établissement

Français du Sang-Alsace,

Strasbourg, France

J.-M. Freyssinet

U770 INSERM, Faculté de Médecine,

Université Paris-Sud, Le Kremlin-Bicêtre, Orsay, France

F. Toti

UMR 7213 CNRS, Faculté de Pharmacie, Université de Strasbourg, Illkirch,

France

Abstract Purpose: Septic shockinduced disseminated intravascular coagulopathy (DIC) contributes to multiple organ failure. Mechanisms governing vascular responses to open occurrence of DIC have not yet been established. Circulating plasma microparticles (MPs), released upon cell stress, constitute a catalytic procoagulant surface and are surrogates of vascular cell activation/injury. Herein, MPs were assessed as possible markers of haemostatic and vascular dysfunction in the DIC time course. Methods: One hundred patients with septic shock from three ICUs were enrolled and their haemostatic status evaluated at admission (D1), D2, D3 and D7. Circulating procoagulant MPs were isolated, quantified by prothrombinase assay and their cellular origin determined. DIC diagnosis was made according to the JAAM 2006 score. Results: Ninety-two patients were analysed and 40 had DIC during the first $24 \mathrm{~h}$. Routine clotting times and factor/inhibitor activity did not allow assessing vascular cell involvement. At admission, thrombin generation and fibrinolysis were observed in both groups while impaired fibrin polymerisation was evidenced only in DIC patients. Sustained thrombin generation persisted over time in both groups at D7. While total microparticle concentrations were in the same range regardless of DIC diagnosis, specific phenotypes were already detected at admission in DIC patients. Endothelial- and leucocyte-derived MPs were higher in DIC while an increased soluble glycoprotein V/platelet ratio was delayed, underscoring the first involvement of endothelial cells and leucocytes whereas platelet activation was delayed. Endotheliumderived CD105-MPs (OR 6.55) and CD31-MPs (OR 0.49) were strongly 
associated with early DIC in multivariate analysis.

Conclusion: Endothelial-derived microparticles are relevant biomark- ers of septic shock-induced DIC and Keywords Septic shock . could be used to evaluate early vascular injury.
Disseminated intravascular coagulopathy · Microparticles . Endothelium $\cdot$ JAAM score

\section{Introduction}

Septic shock is a life-threatening disorder with a host inflammatory response leading to multiple organ dysfunction syndrome (MODS) [1]. Coagulation activation has dual consequences with bacterial containment and thrombotic microangiopathy, eventually evolving to disseminated intravascular coagulopathy (DIC), which is frequently associated with MODS [2, 3]. Furthermore, assessment of both inflammation and haemostasis remains difficult because of major disturbances in regulatory loops [4]. According to a recent review by Gando et al. [5], the DIC paradigm can be viewed as follows: "the blood of patients with DIC is hypercoagulable in the circulation and difficult to clot outside the vessels".

In clinical settings, the mechanisms governing vascular responses and the occurrence/resolution of DIC have not yet been deciphered. There are currently two coexisting DIC scoring systems, one from the International Society for Thrombosis and Haemostasis (ISTH overt 2001) [6] and the other from the Japanese Association for Acute Medicine (JAAM 2006) [7]; both were evaluated in critically ill patients [8-10].

The endothelium plays a central role in the pathophysiology of septic shock contributing to disseminated inflammation and coagulation [11]. Interplay among the neutrophils, endothelium, monocytes and platelets leading to endothelial injury and microvascular thrombosis is well established in the pathogenesis of both DIC and systemic inflammation and finally MODS [4].

Microparticles (MPs) are plasma membrane submicron fragments with procoagulant properties released from stressed cells. They disseminate membrane and cytoplasmic bioactive molecules from parental to neighbouring cells $[12,13]$. MP procoagulant activity is linked to phosphatidylserine (PhtdSer), an aminophospholipid contributing to additional catalytic surface for the assembly of vitamin K-dependent blood coagulation factors and to the eventual presence of tissue factor (TF). Elevated levels of MPs were first associated with thrombotic disorders and further detected as indicators of vascular stress including coagulopathy, vascular occlusion, immune evasion and infection $[14,15]$. Circulating MPs have been reported during septic shock in several clinical studies [16-18] and in experimental animal models $[19,20]$ where MPs could behave as pathogenic markers by providing procoagulant surfaces and by acting as inflammatory vascular mediators [21].

The present study was designed to assess phenotypic and pathophysiological implications of procoagulant PhtdSer-bearing MPs as strong biomarkers of septic shock-induced vascular dysfunction and DIC.

\section{Patients and methods}

\section{Patients}

One hundred consecutive adult patients (18-85 years old) referred for septic shock [22] and treated with norepinephrine and/or epinephrine were prospectively enrolled after admission in medical intensive care units (ICU) from three tertiary hospitals. End-stage heart (NYHA class IV), liver (Child-Pugh classification C) or evolving cancerous diseases were excluded. The Strasbourg University Hospital Ethics Committee approved this multicentre study. Informed consent was obtained from the patient or relatives at admission and confirmed by the patient. Care was provided without a specific therapeutic intervention (Table 1 and supplementary data).

Blood collection and laboratory analysis

Sampling was performed as soon as possible after patients fulfilled septic shock criteria to avoid therapeutic bias (D1) and thereafter on the following mornings (days 2, 3 and 7). Platelet poor plasma (PPP) was obtained after two centrifugations at $2,500 \times g$ for $15 \mathrm{~min}$ and samples were frozen at $-80^{\circ} \mathrm{C}$. Haemostasis was analysed on STA-R ${ }^{\circledR}$ Evolution (Stago) with standard commercial reagents (see supplementary data). Platelet and leucocyte counts were measured daily. Tissue-type plasminogen activator (tPA), plasminogen activator inhibitor-1 (PAI-1), interleukin-6 (IL-6), IL-10, TNF- $\alpha$, monocyte chemotactic protein-1 (MCP-1), sP-selectin and sE-selectin were quantified by ELISA-derived FlowCytomix ${ }^{\mathrm{TM}}$ beads (Bender MedSystems GmbH, Vienna, Austria). Soluble platelet glycoprotein V (sGPV) was quantified by ELISA (Stago). 
Table 1 Patient characteristics

\begin{tabular}{|c|c|c|c|c|}
\hline & $\begin{array}{l}\text { Total } \\
(N=92)\end{array}$ & $\begin{array}{l}\text { No DIC } \\
(N=52)\end{array}$ & $\begin{array}{l}\mathrm{DIC} \\
(N=40)\end{array}$ & $p$ value \\
\hline \multicolumn{5}{|l|}{ Characteristics } \\
\hline Male sex, $N(\%)$ & $59(64.1)$ & $33(63.5)$ & $26(65.0)$ & 0.97 \\
\hline Age, years & $61.5 \pm 15.0$ & $62.3 \pm 14.4$ & $60.5 \pm 15.9$ & 0.58 \\
\hline SÄPS II & $57.5 \pm 18.1$ & $51.9 \pm 14.3$ & $64.9 \pm 20.0$ & $<0.01$ \\
\hline Mortality day $7, N(\%)$ & $15(16.3)$ & $5(9.6)$ & $10(25.0)$ & 0.09 \\
\hline Mortality day $28, N(\%)$ & $27(29.3)$ & $15(28.8)$ & $12(30.0)$ & 0.91 \\
\hline \multicolumn{5}{|l|}{ Organ failure } \\
\hline SOFA at admission & $9.9 \pm 3.0$ & $8.5 \pm 2.1$ & $11.8 \pm 3.0$ & $<0.01$ \\
\hline Respiratory failure, $N(\%)$ & $85(92.4)$ & $46(88.5)$ & $39(97.5)$ & 0.23 \\
\hline Acute renal failure, $N(\%)$ & $52(56.5)$ & $19(36.5)$ & $33(82.5)$ & $<0.01$ \\
\hline Hepatic failure, $N(\%)$ & $7(7.6)$ & $2(3.8)$ & $5(12.5)$ & 0.24 \\
\hline Coma & $23(25.0)$ & $10(19.2)$ & $13(32.5)$ & 0.22 \\
\hline \multicolumn{4}{|l|}{ Site of infection } & $<0.01$ \\
\hline Pneumonia & $49(53.3)$ & $33(63.5)$ & $16(40.0)$ & 0.03 \\
\hline Urinary & $13(14.1)$ & $7(13.5)$ & $6(15.0)$ & 0.83 \\
\hline Abdominal & $13(14.1)$ & $8(15.4)$ & $5(12.5)$ & 0.69 \\
\hline Bacteraemia & $9(9.8)$ & $1(1.9)$ & $8(20.0)$ & 0.01 \\
\hline Unknown & $12(13.3)$ & $3(5.8)$ & $9(22.5)$ & 0.03 \\
\hline \multicolumn{4}{|l|}{ Microorganism } & 0.50 \\
\hline Gram-positive cocci & $27(29.3)$ & $11(21.2)$ & $16(40.0)$ & \\
\hline Enterobacteriae & $20(21.7)$ & $12(23.1)$ & $8(20.0)$ & \\
\hline Other gram-negative bacilli & $13(14.1)$ & $8(15.4)$ & $5(12.5)$ & \\
\hline Unknown & $32(34.8)$ & $21(40.4)$ & $11(27.5)$ & \\
\hline \multicolumn{5}{|l|}{ Therapy } \\
\hline Renal replacement therapy, $N(\%)$ & $35(38.0)$ & $13(25.0)$ & $22(55.0)$ & $<0.01$ \\
\hline Norepinephrine, $N(\%)$ & $91(98.9)$ & $52(100.0)$ & $39(97.5)$ & 0.99 \\
\hline Epinephrine, $N(\%)$ & $7(7.6)$ & $2(3.8)$ & $5(12.5)$ & 0.25 \\
\hline Dobutamine, $N(\%)$ & $21(22.8)$ & $9(17.3)$ & $12(30.0)$ & 0.15 \\
\hline Fresh frozen plasma, $N(\%)$ & $22(23.9)$ & $4(7.7)$ & $18(45.0)$ & $<0.01$ \\
\hline Platelet concentrate, $N(\%)$ & $9(9.8)$ & $1(1.9)$ & $8(20.0)$ & 0.01 \\
\hline Heparin, $N(\%)$ & $54(58.7)$ & $35(67.3)$ & $19(47.5)$ & 0.09 \\
\hline Drotrecogin alfa [activated], $N(\%)$ & $16(17.4)$ & $3(5.8)$ & $13(32.5)$ & $<0.01$ \\
\hline
\end{tabular}

Bold $p$ values refer to the global assessment of differences among a group of parameters

DIC scoring

DIC scores were calculated according to the ISTH 2001 "overt" and JAAM 2006 [6, 7]. Early DIC was confirmed if the JAAM (based on a dynamic evaluation of platelet count and taking into account the inflammatory response in critically ill patients) score was 4 or higher during the first $24 \mathrm{~h}$ at admission (D1) and/or at D2.

Microparticle analysis

cProcoagulant MPs were measured by prothrombinase assay and the results were expressed as nanomolar phosphatidylserine equivalents (nM eq. PhtdSer) (see supplementary data) [23]. The MP phenotype was determined using biotinylated relevant monoclonal antibodies instead of annexin-5: anti-GPIb (platelets), anti-CD11a (leucocytes), anti-CD62E (E-selectin, stimulated endothelial cells), anti-CD31 (apoptotic endothelial cells) and anti-CD105 (stimulated endothelial cells). The normal range in healthy volunteers was previously established in our laboratory [24].
Statistical analysis

Categorical variables were described as frequency, and comparison was performed by the $\chi^{2}$ test or Fisher's exact test. Quantitative data were expressed as mean and standard deviation and analysed with the non-parametric KruskalWallis test. Repeated measures were analysed with ANOVA. When indicated, a linear mixed model and twoway ANOVA including post hoc analyses were performed using a $t$ test with Bonferroni correction for multiple comparisons. Prior to performing this analysis, variables were assessed for normality and non-normally distributed variables were transformed using logarithmic, square root, inverse or exponential transformations. Multiple logistic regression models, which excluded JAAM-related parameters, were performed to explain the occurrence of DIC at D1. Cohen's kappa coefficient was used to measure inter-rater agreement between JAAM 2006 and ISTH 2001 overt DIC scores. All statistics were performed with the MedCalc ${ }^{\mathrm{TM}}$ software, version 12.5.0 (Ostend, Belgium). $p<0.05$ was considered statistically significant. For data presentation in figures, the non-parametrically distributed variables are presented as median values with interquartile range. 


\section{Results}

Patients

Main characteristics of the 92 analysed patients are summarised in Table 1. Eight patients were excluded (six for missing or distorted samples and two for unknown exclusion criteria at admission). All patients fulfilled septic shock criteria at admission. We did not find differences in care and treatments between the centres. According to the JAAM 2006 score, 40 patients (44\%) met the early DIC criteria (30 at D1 and 10 at D2). Of note, Cohen's kappa coefficient between the JAAM 2006 and ISTH scores was 0.71 (good) and 34 patients with early DIC were diagnosed by both scoring systems. Patients with early DIC were more severely ill with higher SAPS2 and SOFA scores at admission $(p<0.01$ and $p<0.001$, respectively). In these latter patients, acute renal failure during the ICU stay was more frequent $(p<0.001)$. At admission (D1), plasma inflammatory markers (IL-6, IL-10, MCP-1, TNF $\alpha$ and CRP) were dramatically increased in all patients $(p<0.05)$ and significantly higher in early DIC $(p<0.05)$ except for TNF $\alpha$ and CRP (see supplementary data). Microorganism genus was not associated with DIC diagnosis $(p=0.50)$ (Table 1). Of note, while pneumonia was less frequent $(p=0.03)$ during DIC, bacteraemia was significantly associated with DIC (among these, four had pneumonia, two had pyelonephritis and one had a catheter infection).
Haemostasis and septic shock

Prothrombin time was increased with subsequent low prothrombin activity occurring to a similar extent in all patients with or without DIC. Although dramatically elevated in DIC patients, D-dimer levels were increased in all patients and remained at a high range until D7. Fibrinogen, a parameter not taken into account by the JAAM 2006 score, was increased in nearly all patients and was $<1 \mathrm{~g} / \mathrm{l}$ in only one patient at admission. JAAM DIC scores remained $<4$ in almost all non-DIC patients $(p=0.61 \mathrm{D} 1-\mathrm{D} 7)$ and were lowered in DIC patients $(p=0.04)$.

Interestingly, an early rise in prothrombin $\mathrm{F} 1+2$ (Fig. 1a) and elevated fibrin monomers (Fig. 1b) were both observed, confirming early thrombin generation and fibrin formation, whereas decreased AT and PC revealed the consumption of natural coagulation inhibitors. In both the non-DIC and DIC groups, sustained thrombin generation and fibrinolysis persisted over time despite supportive care and restored coagulation factors and inhibitors. Nevertheless, delayed AT and PC restoration was observed in DIC patients $(p<0.05$ for time course D1-D7) (see supplementary data). Moreover, the PAI-1 plasma concentration was not significantly different in DIC and returned to baseline at D7 $(p<0.05)$ with delayed improvement when DIC was present (Fig. 1c). Truly soluble E-selectin was elevated in both groups and at the upper range during DIC $(p=0.02)$, highlighting endothelial stress (Fig. 1d).
Fig. 1 Time-course of thrombin and fibrin formation, fibrinolysis and selectins in DIC and non-DIC patients. Results are shown in white (no DIC) or grey (early DIC) whisker boxes (horizontal line inside the box median, upper and lower box limits 25-75th percentiles and T-bars 10-90th percentiles, respectively). Dashed lines correspond to reference values. Statistical analyses represent differences of repeated measures between patients with early or without DIC (DIC vs. no DIC) and differences of time-dependent evolution during ICU stay (days 1, 3 and 7 when available). a Prothrombin fragments $1+2$; b fibrin monomers; c plasminogen activator inhibitor-1 (PAI-1); d soluble E-selectin
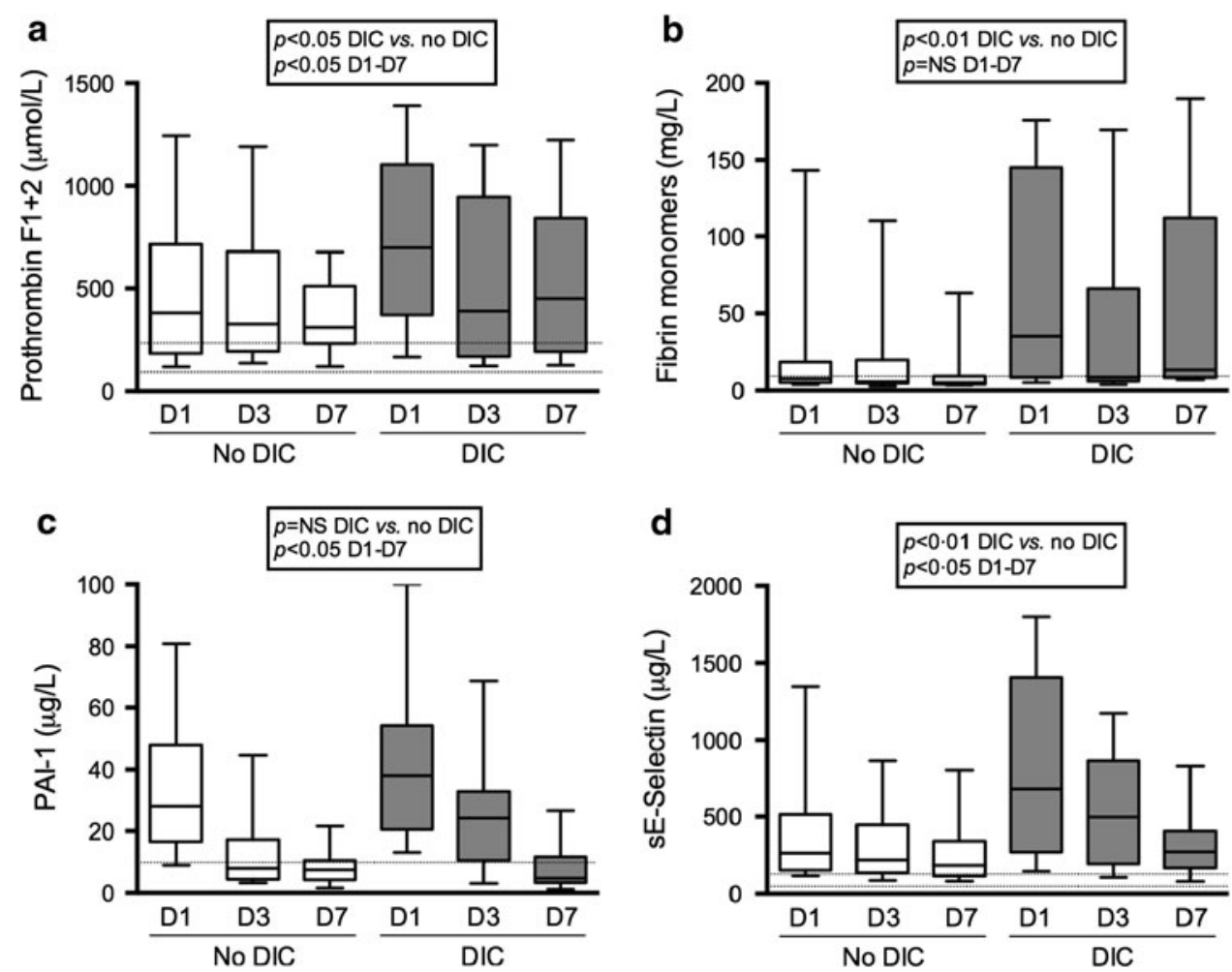
Fig. 2 Time course of total and platelet-, leucocyte- and endothelial cell-derived microparticles regarding DIC diagnosis. Results are shown in white (no DIC) or grey (early DIC) whisker boxes (horizontal line inside box median, upper and lower box limits 25-75th percentiles and T-bars 10-90th percentiles, respectively). Dashed lines correspond to reference values. Statistical analyses represent differences of repeated measures between patients with early or without DIC (DIC vs. no DIC) and differences of time-dependent evolution during the ICU stay (days 1, 3 and 7 when available). Results are in nanomolar equivalent phosphatidylserine (nM eq. PhtdSer) by reference to a standard curve established with liposomes of known composition and concentration. a Total microparticles (PhtdSerMPs); b platelet-derived GPIbMPs to platelet count ratio (GPIb-MPs/platelet); c leucocyte-derived CD11aMPs; d leucocyte-derived CD11a-MPs to leucocyte ratio (CD11a-MPs/Leucocytes); e endothelial cell-derived CD105-MPs; f endothelial cellderived CD31-MPs
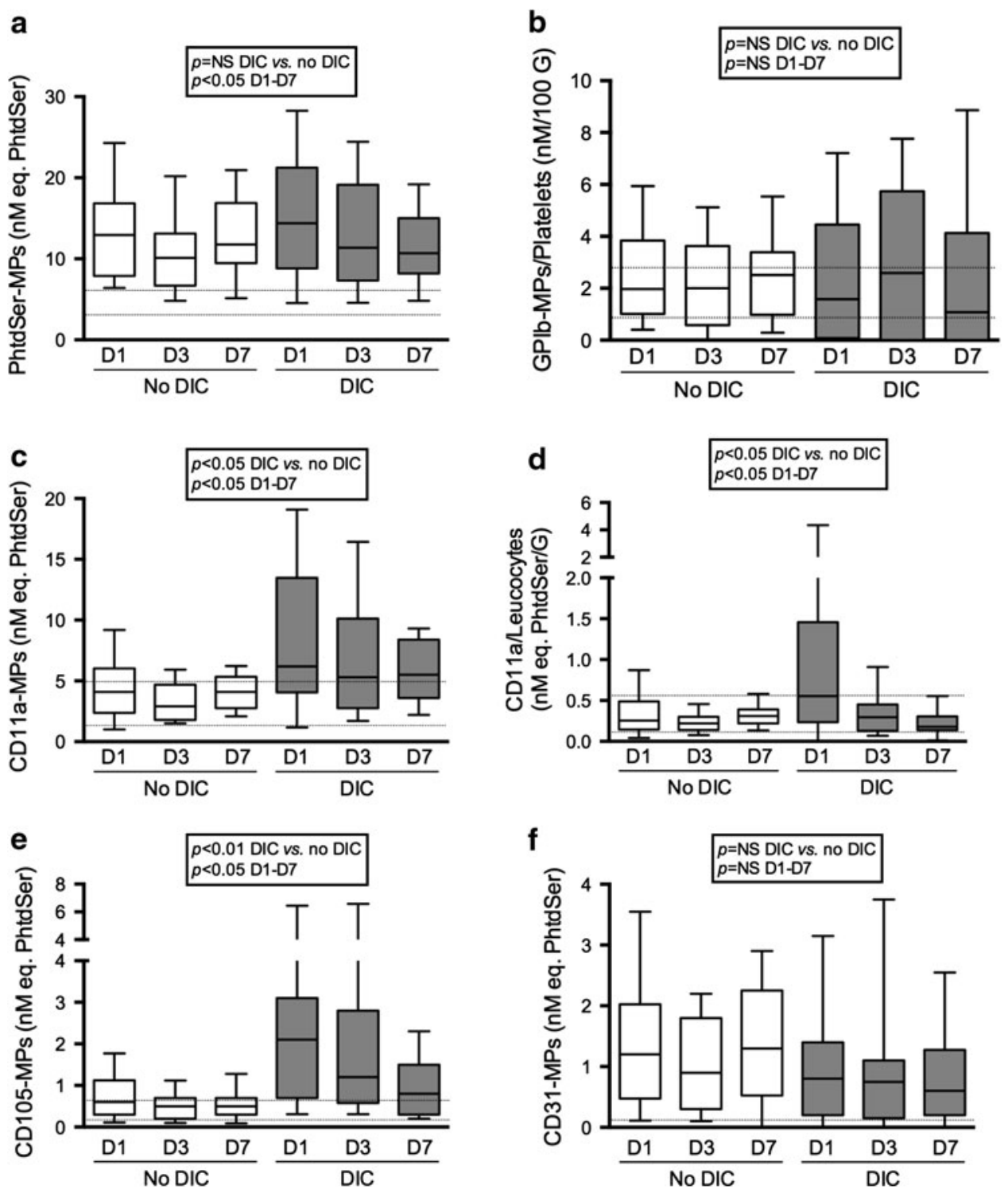

DIC is characterised by a specific microparticle pattern

Total MPs were in the same range in DIC and non-DIC patients but followed a different time-course pattern (Fig. 2a). Nevertheless, compared to non-DIC patients, DIC patients exhibited a specific MP pattern. Circulating GPIb-MPs were lower in DIC ( $p=0.02$ vs. no DIC) (Fig. 2b). Regardless of the DIC diagnosis, MP release was directly dependent on the platelet concentration $\left(r^{2}=0.24, p<0.001\right)$, while the GPIb-MPs/platelet ratio remained stable within the normal range over time (approximately $2.5 \mathrm{nM} / 100 \mathrm{G}$ ).

At admission, CD11a-MP shedding, reflecting leucocyte activation, was drastically increased in the DIC group $(9.2 \pm 8.7$ vs. $4.5 \pm 2.8 \mathrm{nM}$ eq. PhtdSer, $p=0.02$, Fig. 2c) while the leucocyte count was similar in both subsets. During the follow-up period, the CD11aMPs/leucocyte count ratio remained constant in patients without DIC $(0.4 \mathrm{nM} / \mathrm{G})$ while decreasing from $1.5 \mathrm{nM} / \mathrm{G}$ to baseline values at D7 in patients with DIC (Fig. 2d). At D7, CD11a-MP levels remained high in DIC despite normalisation of leucocyte activation with concomitant hyperleucocytosis ( $p<0.05$ vs. no DIC).

Compared to non-DIC patients, CD105-MPs were dramatically increased $(p<0.001)$ in DIC patients and tended to return to baseline at the end of the follow-up period ( $p<0.05$ ) (Fig. 2e). CD31-MPs, which are more indicative of endothelial cell apoptosis, were increased to the same extent in DIC and non-DIC patients, and remained high at D7 (Fig. 2f). Nevertheless, one-way analysis showed that CD31-MPs were significantly lower in DIC patients at D1 $(1.1 \pm 1.2$ vs. $1.6 \pm 2.0 \mathrm{nM}$ eq. PhtdSer, $p<0.001)$. Interestingly, compared to untreated 
DIC patients, those treated by activated protein $\mathrm{C}$ [drotrecogin alfa (activated)] exhibited reduced circulating levels of CD105-MPs $(4.0 \pm 3.8$ vs. $1.3 \pm 1.1 \mathrm{nM}$ eq. PhtdSer, $p<0.001)$ and CD31-MPs $(1.4 \pm 1.9$ vs. $1.0 \pm 1.3 \mathrm{nM}$ eq. PhtdSer, $p=0.06)$ at D3. Fresh frozen plasma, platelet concentrate, unfractionated or low molecular weight heparin and renal replacement therapy did not significantly alter leucocyte- and endothelial cellderived MPs (data not shown). Of note, ISTH DIC scoring did not impact these results (see supplementary data).

The time course of cell activation during septic shockinduced DIC

Patients who met the DIC criteria during the first $24 \mathrm{~h}$ (DIC at D2) were characterised by a mixed pattern at admission. They had moderately increased D-dimers in the same range as non-DIC patients (Fig. 3a). The platelet count was lower than that observed in non-DIC patients, but still above the cutoff value of JAAM (or ISTH) DIC scores (Fig. 3b). Soluble GPV (sGPV), assessed as another indicator of platelet activation by thrombin, confirmed low platelet activation with a sGPV/platelet ratio in the normal range (Fig. 3c). Nevertheless, compared to non-DIC patients, patients who met the DIC criteria at D2 presented an enhanced inflammatory burst (see supplementary data) with increased thrombin generation (prothrombin $\mathrm{F} 1+2$, Fig. 3d) and endothelial cell (CD105-MPs and sEselectin) and leucocyte activation ( $p<0.05$ vs. no DIC, Fig. 3e, f and see supplementary data). At D3, these patients clearly had DIC, with increased D-dimers and a low platelet count, and platelet activation was obvious (Fig. 3a-c). Endothelial cells and leucocytes still remained activated (Fig. 3e, f).

\section{Microparticles may help towards early DIC} assessment

Relevant biological parameters at D1 not related to the JAAM score were challenged in a logistic regression analysis in order to explain early DIC occurrence.
Fig. 3 Time course of cell activation and haemostasis activation during septic shock. Results are shown in white (no DIC), light grey (meeting DIC criteria at D2) or grey (DIC) whisker boxes (horizontal line inside box median, upper and lower box limits 25-75th percentiles and T-bars 10-90th percentiles, respectively). Dashed lines correspond to reference values. Statistical analyses represent differences at admission (D1) between patients without DIC or with DIC at day 1 (admission) or at day 2 (during the first $24 \mathrm{~h}$ of care), $* p<0.05$ no DIC versus meeting DIC criteria at D2; ${ }^{\#} p<0.05$ no DIC versus DIC; $\$ p<0.05$ DIC versus meeting DIC criteria at D2. a D-dimers; b platelet count; c soluble platelet glycoprotein V/platelets ratio (sGPV/platelets); d prothrombin $\mathrm{F} 1+2$; e endothelial cell-derived CD105-MPs; f leucocytederived CD11a-MP to leucocyte ratio (CD11a-MPs/leucocytes)
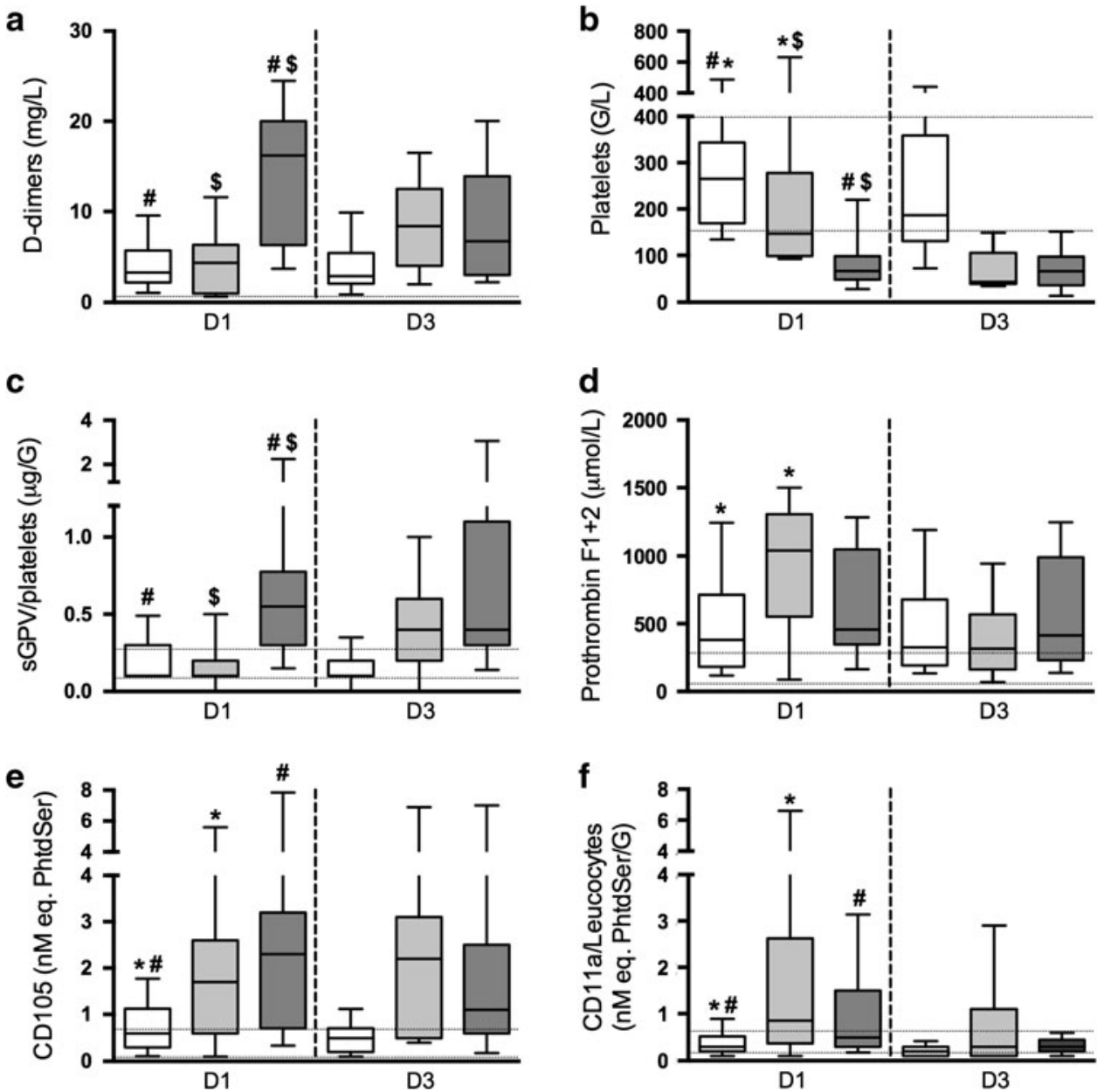
Table 2 Multiple logistic regression at admission (early DIC vs. no DIC)

\begin{tabular}{lllr}
\hline Parameter & Odds-ratio & $\begin{array}{l}95 \% \text { confidence } \\
\text { interval }\end{array}$ & $p$ value \\
\hline CD105-MPs & 6.55 & {$[2.54-16.92]$} & $<0.01$ \\
CD31-MPs & 0.49 & {$[0.26-0.94]$} & 0.03 \\
Factor V & 0.97 & {$[0.95-0.99]$} & 0.02 \\
Fibrin monomers & 1.01 & {$[1.00-1.02]$} & 0.06 \\
$\begin{array}{l}\text { Prothrombin } \\
\quad \text { fragment 1+2 }\end{array}$ & 1.00 & {$[1.00-1.00]$} & 0.09 \\
\hline
\end{tabular}

PAI-1, AT, PC, sE-selectin, sP-selectin, CD11a-MPs/leucocyte ratio, IL-6, IL-10, MCP-1 and TNF $\alpha$ were not significantly associated with DIC

CD105-MPs, CD31-MPs, factor V, prothrombin F1+2 and fibrin monomers were associated with DIC (Table 2). In this model, PAI-1, AT, PC, sE-selectin, sP-selectin, CD11a-MPs/leucocyte ratio, IL-6, IL-10, MCP-1 and TNF $\alpha$ were not significantly associated with DIC.

\section{Discussion}

In septic shock, vascular injury is central and is prompted by different actors with overlapping kinetics, leading to difficulties in the deciphering of a sequential order [25]. Despite biological criteria and scores, ultimate disseminated intravascular coagulopathy is difficult to assess clinically $[26,27]$. As the inflammatory cascade represents the main limitation of the ISTH overt score, the JAAM score could be more helpful in DIC detection [9, 28, 29]. Nevertheless, we found that at D1, DIC scoring had no effect on the outcome of the analysis of the variation in biomarkers, including MPs.

According to the DIC scoring criteria, we report a high incidence of "early" DIC diagnosed during the first $24 \mathrm{~h}$ and encompassing about $40 \%$ of patients with septic shock. As previously reported, early DIC is strongly associated with septic shock severity (SAPS II) and organ failure (SOFA), but not with mortality [30]. From the present standpoint of haemostatic activation/deregulation during sepsis, one could consider a more dynamic imbalance evolving over time in patients meeting DIC criteria. Thus, the present prospective study was designed to evaluate the association between procoagulant MPs and DIC occurrence during the time course of septic shock. Our data confirm specific vascular damage whereby endothelial cell and leucocyte impairment subsequently evolves to deregulated thrombin generation and to DIC, despite therapeutic intervention. In a cell-based model of haemostasis, DIC may not only be viewed as a coagulation disorder, but also as a delayed symptom of emerging systemic vascular inflammatory disease $[2,4]$.
The possibility of MP-driven coagulation is supported by the reported role of MPs in meningococcemia [31] or by the dissemination of a procoagulant signal to neutrophils and platelets [32]. Our data evidenced elevated levels of total MPs in septic patients and a concomitant rise in prothrombin $\mathrm{F} 1+2$, which is highly suggestive of an MP-driven activated coagulation pathway. Indeed, PhtdSer-MPs constitute an additional catalytic surface for blood coagulation that could partially compensate for the low platelet count observed during DIC. A recent report of thrombin generation triggered by platelet-MPs in an FVII-independent but FXII-dependent manner [33] raises the likelihood of a particular platelet contribution in increasing thrombin generation. Our observation of delayed platelet activation may reflect the presence of thrombin as the main agonist while GPVI activation by sub-endothelial collagen is not predominant [34].

In view of the present data, MPs appear to behave as vascular effectors in the course of sepsis and DIC. Multivariate analysis of the MP phenotype at D1 evidenced a particular pattern that indicates an evolution to DIC whereas routine laboratory tests failed to identify patients with an "intermediate" platelet count range and elevated prothrombin $\mathrm{F} 1+2$. Furthermore, as platelet activation is not yet detectable by sGPV/platelet and sP-selectin/ platelet ratios, which remain low at D1, the MP phenotype pattern appears to be more informative with a characteristic drop in platelet-derived GPIb-MPs following a drop in platelets and a rise in leucocyte-derived CD11a-MPs and endothelial-derived CD105-MPs. Altogether, data from the present and other studies tend to draw a picture of DIC genesis during septic shock supported by endothelial injury after an inflammatory burst, with leucocyte recruitment [25]. Indeed, our data confirm that the very initial high levels of cytokines and chemokines prompt endothelial and leucocyte activation prior to septic shock-induced DIC [35].

Our study also indicates that MPs are a valuable tool for the detection of late cell events in the time course of sepsis. We evidenced an original specific drop in CD105MPs values, which returned to normal range at D7 in DIC patients. These MPs could prove to be a better indicator than CD31-MPs in the monitoring of endothelial damagedriven DIC over time.

According to the Bradford-Hill criteria, causation requires temporality. From the present standpoint, endothelial and leucocyte activation precedes DIC diagnosis with CD105-MPs appearing to be the best predictor [36]. Furthermore, microparticle clearance from plasma is not clearly demonstrated, although their pharmacological modulation could constitute a reasonable challenge [18, 37], while the implication of CD105 is of interest in the context of septic shock-induced DIC. Of note, patients treated in the present study with drotrecogin alfa (activated), known to have cytoprotective effects [38], had significantly lowered CD105-MPs at D3. 
Finally, we highlight that routine haemostatic assays could be combined with MPs as indicators of endothelial cell and leucocyte involvement during septic shock. Using this approach, three groups of patients as opposed to two could be identified: (1) patients with low-grade vascular injury and controlled thrombin generation (no DIC), (2) patients with inflammatory burst, endothelial cell and leucocytes activation but with still-controlled thrombin generation who meet the DIC criteria at D2 (only 10 patients in our cohort) and (3) patients with endothelial and leucocyte activation resulting in deregulated thrombin generation and high-level fibrinolysis who meet the DIC criteria. We suggest that our preliminary results of MP patterns could help clinicians to improve early DIC assessment in order to allow a better stratification of patients treated for septic shock in future clinical trials. Thus, a new cohort is warranted to establish a new combined cell-based and routine clotting time score to diagnose early vascular injury during septic shock.

Acknowledgments XD, FT and FM had full access to all the study data and take responsibility for their integrity and the accuracy of the analysis. XD, FT, JMF and FM participated in the study design and obtained funding for the study. XD, PA, YM, JHB, AB and FM collected the data. XD, TL, FT and FM analysed the data; TL provided statistical expertise. XD, JHB, AB, LG, FL and FT performed biological analyses. XD, FT, FL and FM drafted the report, and the report was revised for important intellectual content by XD, PA, LG, CG, JMF, FT and FM. All authors read and approved the final manuscript.

We would like to thank Fatiha Zobairi for sample preparation and MP measurements, Jacqueline Picard for routine haemostasis tests, Sylvie Moog for sGPV analysis, François Lefebvre (MD) for complementary statistical analysis and Maleka Schenck-Dhif (MD) and Dominique Desprez (MD) for manuscript revision and Pierre Pothier for careful English proofreading of the manuscript.

This work was supported by grants from the French Ministry of Health (Projet Hospitalier de Recherche Clinique, Hôpitaux Universitaires de Strasbourg no. 4649), Laboratoire Français de Fractionnement et des Biotechnologies (LFB SA, Les Ullis, France), the Société de Réanimation de Langue Française (SRLF, Paris, France) and the Association pour le Développement et la Recherche en Réanimation (ADRER, Strasbourg, France).

Conflicts of interest The authors declare that they have no conflicts of interest.

\section{References}

1. Annane D, Bellissant E, Cavaillon JM (2005) Septic shock. Lancet 365:63-78

2. Fourrier F (2012) Severe sepsis, coagulation, and fibrinolysis: dead end or one way? Crit Care Med 40:2704-2708

3. Levi M (2012) Coagulation and sepsis: a winding road ahead. Crit Care Med 40:2733-2734

4. Gando S (2010) Microvascular thrombosis and multiple organ dysfunction syndrome. Crit Care Med 38:S35-S42

5. Gando S, Wada H, Tachil J; The Scientific Standardization Committee on DIC of the International Society on Thrombosis and Haemostasis (ISTH) (2013) Differentiating disseminated intravascular coagulation (DIC) with the fibrinolytic phenotype from coagulopathy of trauma and acute coagulopathy of trauma-shock (COT/ ACOTS). J Thromb Haemost 11:826-835

6. Taylor FB Jr, Toh CH, Hoots WK, Wada H, Levi M (2001) Towards definition, clinical and laboratory criteria, and a scoring system for disseminated intravascular coagulation. Thromb Haemost 86:1327-1330
7. Gando S, Iba T, Eguchi Y, Ohtomo Y, Okamoto K, Koseki K, Mayumi T, Murata A, Ikeda T, Ishikura H, Ueyama M, Ogura H, Kushimoto S, Saitoh D, Endo S, Shimazaki S (2006) A multicenter, prospective validation of disseminated intravascular coagulation diagnostic criteria for critically ill patients: comparing current criteria. Crit Care Med 34:625-631

8. Bakhtiari K, Meijers JC, de Jonge E, Levi M (2004) Prospective validation of the International Society of Thrombosis and Haemostasis scoring system for disseminated intravascular coagulation. Crit Care Med 32:2416-2421

9. Singh RK, Baronia AK, Sahoo JN, Sharma S, Naval R, Pandey CM, Poddar B, Azim A, Gurjar M (2012) Prospective comparison of new Japanese Association for Acute Medicine (JAAM) DIC and International Society of Thrombosis and Hemostasis (ISTH) DIC score in critically ill septic patients. Thromb Res 129:e119-e125

10. Wada H, Thachil J, Di Nisio M, Mathew P, Kurosawa S, Gando S, Kim HK, Nielsen JD, Dempfle CE, Levi M, Toh CH; The Scientific Standardization Committee on DIC of the International Society on Thrombosis and Haemostasis (2013) Guidance for diagnosis and treatment of DIC from harmonization of the recommendations from three guidelines. $\mathbf{J}$ Thromb Haemost 11:761-767
11. Boisramé-Helms J, Kremer H, SchiniKerth V, Meziani F (2013) Endothelial dysfunction in sepsis. Curr Vasc Pharmacol 11:150-160

12. Meziani F, Tesse A, Andriantsitohaina R (2008) Microparticles are vectors of paradoxical information in vascular cells including the endothelium: role in health and diseases. Pharmacol Rep 60:75-84

13. Mause SF, Weber C (2010) Microparticles: protagonists of a novel communication network for intercellular information exchange. Circ Res 107:1047-1057

14. Meziani F, Delabranche X, Asfar P, Toti F (2010) Bench-to-bedside review: circulating microparticles a new player in sepsis? Crit Care 14:236

15. Delabranche X, Berger A, BoisraméHelms J, Meziani F (2012)

Microparticles and infectious diseases. Med Mal Infect 42:335-343

16. Schouten M, Wiersinga WJ, Levi M, van der Poll T (2008) Inflammation, endothelium, and coagulation in sepsis. J Leukoc Biol 83:536-545

17. Joop K, Berckmans RJ, Nieuwland R, Berkhout J, Romijn FP, Hack CE, Sturk A (2001) Microparticles from patients with multiple organ dysfunction syndrome and sepsis support coagulation through multiple mechanisms. Thromb Haemost $85: 810-820$ 
18. Perez-Casal M, Thompson V, Downey C, Welters I, Wyncoll D, Thachil J, Toh $\mathrm{CH}$ (2011) The clinical and functional relevance of microparticles induced by activated protein $C$ treatment in sepsis. Crit Care 15:R195

19. Mortaza S, Martinez MC, BaronMenguy C, Burban M, De la Bourdonnaye M, Fizanne L, Pierrot M, Cales P, Henrion D, Andriantsitohaina R, Mercat A, Asfar P, Meziani F (2009) Detrimental hemodynamic and inflammatory effects of microparticles originating from septic rats. Crit Care Med 37:2045-2050

20. Zafrani L, Gerotziafas G, Byrnes C, Hu X, Perez J, Levi C, Placier S, Letavernier E, Leelahavanichkul A, Haymann JP, Elalamy I, Miller JL, Star RA, Yuen PS, Baud L (2012) Calpastatin controls polymicrobial sepsis by limiting procoagulant microparticle release. Am J Respir Crit Care Med 185:744-755

21. Reid VL, Webster NR (2012) Role of microparticles in sepsis. Br J Anaesth 109:503-513

22. Levy MM, Fink MP, Marshall JC, Abraham E, Angus D, Cook D, Cohen J, Opal SM, Vincent JL, Ramsay G (2003) 2001 SCCM/ESICM/ACCP/ ATS/SIS international sepsis definitions conference. Intensive Care Med 29:530-538

23. Hugel B, Zobairi F, Freyssinet JM (2004) Measuring circulating cellderived microparticles. J Thromb Haemost 2:1846-1847

24. Bakouboula B, Morel O, Faure A, Zobairi F, Jesel L, Trinh A, Zupan M, Canuet M, Grunebaum L, Brunette A, Desprez D, Chabot F, Weitzenblum E, Freyssinet JM, Chaouat A, Toti F (2008) Procoagulant membrane microparticles correlate with the severity of pulmonary arterial hypertension. Am J Respir Crit Care Med 177:536-543
25. Shapiro NI, Schuetz P, Yano K, Sorasaki M, Parikh SM, Jones AE, Trzeciak S, Ngo L, Aird WC (2010) The association of endothelial cell signaling, severity of illness, and organ dysfunction in sepsis. Crit Care 14:13

26. Levi M, de Jonge E, van der Poll $\mathrm{T}$ (2003) Sepsis and disseminated intravascular coagulation. J Thromb Thrombolysis 16:43-47

27. Levi M (2010) The coagulant response in sepsis and inflammation. Hamostaseologie 30(10-12):14-16

28. Kushimoto S, Gando S, Saitoh D, Ogura H, Mayumi T, Koseki K, Ikeda $\mathrm{T}$, Ishikura $\mathrm{H}$, Iba $\mathrm{T}$, Ueyama $\mathrm{M}$, Eguchi Y, Otomo Y, Okamoto K, Endo S, Shimazaki S (2008) Clinical course and outcome of disseminated intravascular coagulation diagnosed by Japanese Association for Acute Medicine criteria. Comparison between sepsis and trauma. Thromb Haemost 100:1099-1105

29. Takemitsu T, Wada H, Hatada T, Ohmori Y, Ishikura K, Takeda T, Sugiyama T, Yamada N, Maruyama K, Katayama N, Isaji S, Shimpo H, Kusunoki M, Nobori T (2011) Prospective evaluation of three different diagnostic criteria for disseminated intravascular coagulation. Thromb Haemost 105:40-44

30. Okabayashi K, Wada H, Ohta S, Shiku H, Nobori T, Maruyama K (2004) Hemostatic markers and the sepsisrelated organ failure assessment score in patients with disseminated intravascular coagulation in an intensive care unit. Am J Hematol $76: 225-229$
31. Nieuwland R, Berckmans RJ, McGregor S, Boing AN, Romijn FP, Westendorp RG, Hack CE, Sturk A (2000) Cellular origin and procoagulant properties of microparticles in meningococcal sepsis. Blood 95:930-935

32. Osterud B (2010) Tissue factor expression in blood cells. Thromb Res 125:S31-S34

33. Van Der Meijden PE, Van Schilfgaarde M, Van Oerle R, Renne T, ten Cate H, Spronk HM (2012) Platelet- and erythrocyte-derived microparticles trigger thrombin generation via factor XIIa. J Thromb Haemost 10:1355-1362

34. Heemskerk JW, Mattheij NJ, Cosemans JM (2013) Platelet-based coagulation: different populations, different functions. J Thromb Haemost 11:2-16

35. de Jong HK, van der Poll T, Wiersinga WJ (2010) The systemic proinflammatory response in sepsis. J Innate Immun 2:422-430

36. Ramma W, Ahmed A (2011) Is inflammation the cause of preeclampsia? Biochem Soc Trans 39:1619-1627

37. Dasgupta SK, Abdel-Monem H, Niravath P, Le A, Bellera RV, Langlois K, Nagata S, Rumbaut RE, Thiagarajan P (2009) Lactadherin and clearance of platelet-derived microvesicles. Blood 113:1332-1339

38. Mosnier LO, Zlokovic BV, Griffin JH (2007) The cytoprotective protein $C$ pathway. Blood 109:3161-3172 